Progress in Flight Physics 5 (2013) 379-392

DOI: $10.1051 /$ eucass/201305379

(C) Owned by the authors, published by EDP Sciences, 2013

\title{
HYPERSONIC BOUNDARY LAYER IN THE VICINITY OF A POINT OF INFLECTION OF LEADING EDGE ON A FLAT WING IN THE REGIME OF STRONG VISCOUS INTERACTION
}

\author{
G. N. Dudin and A. V. Ledovskiy \\ Moscow Institute of Physics and Technology \\ Moscow, Russia
}

\begin{abstract}
The flow in a spatial hypersonic laminar boundary layer on a planar wing with a point of inflection in the leading edge is considered in the regime of strong viscous-inviscid interaction. The boundary problems are formulated for two cases: self-similar flow near the point of inflection of the leading edge and full three-dimensional (3D) boundary layer on a wing with variable sweep angle. The numerical solution is obtained using the finite-difference method. The results of parametric calculations of influence of a wing shape and the temperature factor on flow characteristics in the boundary layer are presented. The possibility of formation of local regions with high shear stress and heat flux is shown.
\end{abstract}

\section{INTRODUCTION}

With development of aeronautics and space flights hypersonic aerodynamics has become an important part of modern fluid dynamics [1]. Due to the high temperature and large gradients of flow characteristics, an experimental study is very difficult. So, the most common method of investigation of such flows is computational fluid dynamics. But because of high Reynolds number and strong shock waves, the solution of full Navier-Stokes equations is a rather complicated task. Thus, solving the boundary layer equations is still actual for theoretical analysis and can give good results for local characteristics such as the surface friction, the heat flux, and the pressure.

This work is devoted to studying the flow in a hypersonic laminar boundary layer on wings with a variable sweep angle of the leading edge in the regime of strong interaction. The method developed can be easily applied to investigation 
of boundary layer on a wing of a complex shape, with sharp leading edges at zero angle of attack. In the present paper some examples for planar wings with different shapes and temperature factors are demonstrated.

\section{MATHEMATICAL FORMULATION OF THE PROBLEM}

\subsection{Self-Similar Equations Near the Point of Inflection of the Leading Edge}

The hypersonic flow around a flat wing is considered with a point of inflection of the leading edge at zero angle of attack with strong viscous interaction under the following conditions:

$$
\mathrm{M}_{\infty} \rightarrow \infty, \quad \operatorname{Re}_{0} \rightarrow \infty, \quad \mathrm{M}_{\infty} \delta \rightarrow \infty
$$

where $M_{\infty}$ is the Mach number of the external inviscid flow; $\operatorname{Re}_{0}=\rho_{\infty} u_{\infty} / \mu_{0}$ is the Reynolds number; and $\delta=\left(\Theta / \operatorname{Re}_{0}\right)^{1 / 4}$ is the dimensionless boundary layer thickness. The parameter $\Theta$ is the angle between the bisector and the edge of the wing. A two-layer flow scheme is used - the outer region of inviscid flow described by the Euler equations, and a viscous laminar boundary layer governed by the Prandtl equations. The gas is assumed to be thermodynamically perfect with a constant specific heat ratio $\gamma$. The Reynolds number is high but not higher than the critical value so that the flow is laminar. The surface temperature is considered to be known and constant over the entire surface of the wing. Basic geometric parameters of the problem are $\Theta$ and $\beta$ - the angle between the external flow and the bisector angle of the wing. A cylindrical coordinate system with the center at the point of inflection of the leading edge (Fig. 1) is introduced.

To determine the external pressure distribution, the approximate "tangent wedge" formula [2] is used. In accordance with the geometrical parameters of the problem and the characteristic parameter estimations for the boundary layer flow in a hypersonic flow $[2,3]$, the dimensionless variables: $L r, \Theta \theta, L \delta y, \Theta \beta, U_{\infty} u$, $U_{\infty} w, U_{\infty} \delta \Theta^{-1} v, U_{\infty}^{2} H / 2, \rho_{\infty} \delta^{2} \rho, \rho_{\infty} U_{\infty}^{2} \delta^{2} p, \mu_{0} \mu$, and $L \delta \delta_{e}$ are introduced. Here, $L$ is the characteristic linear dimension; $H$ is the total enthalpy; $\mu_{0}$ is the dynamic viscosity coefficient at the stagnation temperature; and the subscript $\infty$ denotes the dimensional parameters of the external flow.

Let introduce new variables in accordance with the transformation of Dorodnitsyn [2]:

$$
\lambda=\int_{0}^{y} \rho d y ; \quad v_{\delta}=\rho v+\frac{w}{r} \frac{\partial \lambda}{\partial \theta}+\Theta u \frac{\partial \lambda}{\partial r} .
$$




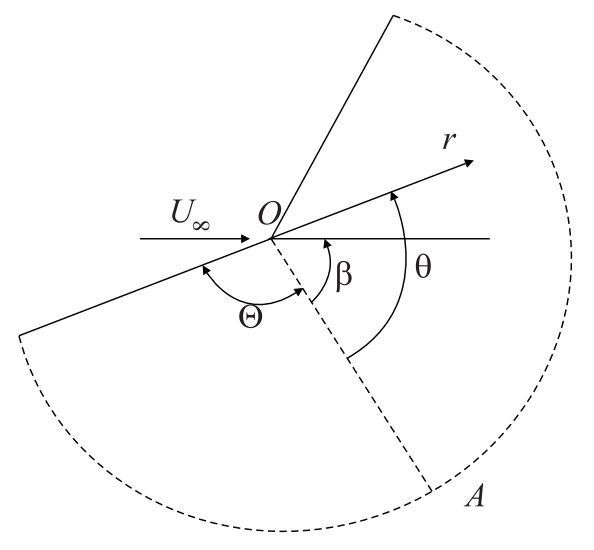

Figure 1 Semiinfinite wing in the vicinity of point of inflection

Then the expression for the displacement thickness of the boundary layer takes the form:

$$
\delta_{e}=\frac{\gamma-1}{2 \gamma p} \int_{0}^{\infty}\left(H-u^{2}-w^{2}\right) d \lambda
$$

For the numerical solution of the boundary problem, the behavior of the functions of the flow near the leading edge $(\theta= \pm 1)$ and at the critical point $(r=0)$ must be taken into account. For this purpose, the following variables which in the case of a semiinfinite wing can reduce the problem to the twodimensional (2D) one, are introduced:

$$
\begin{aligned}
\eta & =\lambda r^{-1 / 4}\left(\frac{2 \gamma}{\gamma-1} \sqrt{1-\theta^{2}}\right)^{-1 / 2} \\
v_{*} & =\left(v_{\delta} r^{3 / 4}+r \Theta u \frac{\partial\left(\lambda r^{-1 / 4}\right)}{\partial r}\right) \frac{\left(1-\theta^{2}\right)^{3 / 4}}{p^{*}} \sqrt{\frac{\gamma-1}{2 \gamma}+\left(1-\theta^{2}\right) \frac{w}{p^{*}} \frac{\partial \eta}{\partial \theta}} \\
p & =p^{*} r^{-1 / 2}\left(1-\theta^{2}\right)^{-1 / 2} ; \\
\rho & =r^{-1 / 2} \rho^{*} \\
\delta_{e} & =r^{3 / 4}\left(1-\theta^{2}\right)^{3 / 4} \Delta
\end{aligned}
$$

Furthermore, it is assumed that the dynamic viscosity coefficient has a power dependence on the temperature $\mu=\left(H-u^{2}-w^{2}\right)^{w}$. As a result of these transformations, the boundary problem takes the form: 


$$
\begin{gathered}
\frac{\partial v_{*}}{\partial \eta}=w \frac{\theta}{2 p^{*}}-\frac{1-\theta^{2}}{p^{*}}\left(\Theta r \frac{\partial u}{\partial r}+\frac{\partial w}{\partial \theta}+\frac{5}{4} \Theta u\right) ; \\
\frac{1-\theta^{2}}{p_{*}}\left(\Theta r u \frac{\partial u}{\partial r}+w \frac{\partial u}{\partial \theta}-\Theta w^{2}\right)+v_{*} \frac{\partial u}{\partial \eta} \\
=\Theta \frac{1-\theta^{2}}{p_{*}} \frac{\gamma-1}{2 \gamma}\left(g-u^{2}-w^{2}\right)\left(\frac{1}{2}-\frac{r}{p_{*}} \frac{\partial p_{*}}{\partial r}\right)+\frac{\partial}{\partial \eta}\left(N \frac{\partial u}{\partial \eta}\right) ; \\
\frac{1-\theta^{2}}{p_{*}}\left(\Theta r u \frac{\partial w}{\partial r}+w \frac{\partial w}{\partial \theta}+\Theta u w\right)+v_{*} \frac{\partial w}{\partial \eta} \\
=-\frac{\gamma-1}{2 \gamma}\left(g-u^{2}-w^{2}\right)\left(\frac{\theta}{p_{*}}+\frac{1-\theta^{2}}{p_{*}^{2}} \frac{\partial p_{*}}{\partial \theta}\right)+\frac{\partial}{\partial \eta}\left(N \frac{\partial w}{\partial \eta}\right) ; \\
\frac{1-\theta^{2}}{p^{*}}\left(\Theta r u \frac{\partial H}{\partial r}+w \frac{\partial H}{\partial \theta}\right)+v_{*} \frac{\partial H}{\partial \eta} \\
\quad \eta=0: \quad \frac{\partial}{\partial \eta}\left(N\left[\frac{1}{\sigma} \frac{\partial H}{\partial \eta}-\frac{1-\sigma}{\sigma} \frac{\partial\left(u^{2}+w^{2}\right)}{\partial \eta}\right]\right) ; \\
N=\left(H-v^{2}-w^{2}\right)^{\omega-1} ; \\
\left.\left.\Delta=\frac{1}{p_{*}} \sqrt{\frac{\gamma-1}{2 \gamma}} \int_{0}^{\infty}(H-\beta)\right]\left(\frac{3}{2} \theta \Delta_{e}-\left(1-\theta^{2}\right) \frac{\partial \Delta_{e}}{\partial \theta}\right)\right\}^{2} ; \\
p_{*}=\frac{\gamma+1}{2}\left\{\left(1-\theta^{2}\right)\left(\frac{3}{4} \Delta_{e}+r \frac{\partial \Delta_{e}}{\partial r}\right) \cos [\Theta(\theta-\beta)]\right.
\end{gathered} ;
$$

The boundary conditions on the surface of wing are no-slip conditions and the surface temperature is constant that is defined as a temperature factor $H_{w}$ (the ratio of the surface temperature to the stagnation temperature). On the outer edge, the external flow is assumed to be uniform.

For a semiinfinite wing, the system of Eqs. (2) can be reduced to self-similar $2 \mathrm{D}$ equations which are identical to equations at $r=0[1,2]$. At the edge of the wing, the term $(1-\theta)^{2}$ becomes zero, and, thus, the system of partial differential equations is reduced to a system of ordinary differential equations. Integrating of the last ones allows to find the velocity profiles and the enthalpy, the pressure, and the displacement thickness at the leading edge, which are used as boundary conditions for $2 \mathrm{D}$ equations. 


\subsection{Flow on the Wings with Variable Form of Leading Edge}

Let consider a symmetric planar wing with a variable sweep angle of the leading edge in a hypersonic flow of a viscid ideal gas. The angle of attack is assumed to be zero. The form of leading edge is set by function $z_{e}(x)$. The parameter $z_{0}$ is a half width of the trailing edge. Here, the cartesian coordinate system is

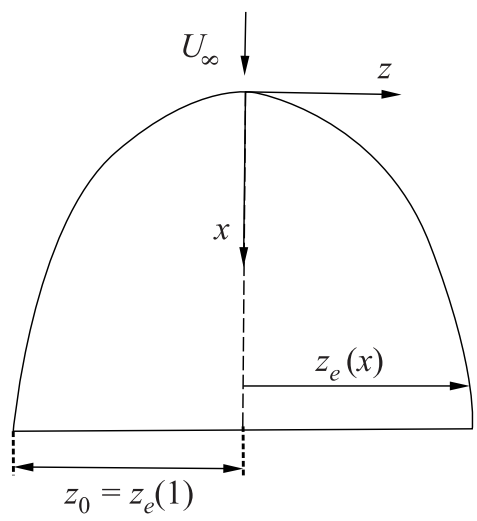

Figure 2 Wing with variable sweep angle of leading edge introduced in such a way that the origin is placed in a vertex of wing, axis $X$ in aligned along thr incoming flow, and axis $Y$ is aligned along the normal to the wing (Fig. 2).

The same assumptions (1) are used. The problem is considered with the assumption that there is a strong viscousinviscid interaction with the interaction parameter [1]:

$$
\chi=\frac{\mathrm{M}_{\infty}^{2}}{\sqrt{\mathrm{Re}_{0}}} \gg 1 .
$$

The functions of the pressure and the boundary layer displacement thickness are well known for this type of flow [2]:

$$
\delta^{*} \sim x^{3 / 4} ; \quad p \sim \frac{1}{\sqrt{x}} .
$$

The problem is considered within the framework of $3 \mathrm{D}$ equations of a laminar compressible boundary layer in a rectangular Cartesian coordinate system. The external pressure distribution is defined by the approximate "tangent wedge" formula that in the regime of strong interaction in Cartesian coordinate system takes the following form:

$$
p=\frac{\gamma+1}{2} \rho_{\infty} U_{\infty}^{2}\left(\frac{\partial \delta}{\partial x}\right)^{2} .
$$

The dimensionless parameters for this case are:

$$
\begin{gathered}
x^{*}=L x ; z^{*}=z_{0} z ; y^{*}=L \delta y \\
u^{*}=U_{\infty} u ; w^{*}=U_{\infty} w ; v^{*}=U_{\infty} \delta z_{0}^{-1} v ; H^{*}=\frac{U_{\infty}^{2} H}{2} \\
\rho^{*}=\rho_{\infty} \delta^{2} \rho ; p^{*}=\rho_{\infty} U_{\infty}^{2} \delta^{2} p ; \quad \mu^{*}=\mu_{0} \mu ; \delta_{e}^{*}=L \delta \delta_{e} .
\end{gathered}
$$

For numerical calculations, some transformations are made, and new variables are introduced: 


$$
\begin{gathered}
\lambda=\int_{0}^{y} \rho d y ; \quad v_{\delta}=\rho v+w \frac{\partial \lambda}{\partial z}+z_{0} u \frac{\partial \lambda}{\partial x} ; \\
\lambda^{*}=x^{-1 / 4} \lambda ; v^{*}=v_{\delta} x^{3 / 4}+z_{0} x u \frac{\partial \lambda^{*}}{\partial x} ; z=z_{e}(x) t ; \\
p=x^{-1 / 2} p^{*}(x, t) ; \rho=x^{-1 / 2} \rho^{*}\left(x, \lambda^{*}, t\right) ; \delta_{e}^{0}=x^{3 / 4} \delta_{e}^{*}(x, t) ; \\
\eta=\lambda^{*}\left(\frac{2 \gamma}{\gamma-1} \sqrt{1-t^{2}}\right)^{-1 / 2} ; \\
v_{*}=v^{*} \frac{\left(1-t^{2}\right)^{3 / 4}}{p^{*}} \sqrt{\frac{\gamma-1}{2 \gamma}+\frac{\left(1-t^{2}\right)}{p^{*}}\left(\frac{w x}{z_{e}}-\frac{z_{0} u t x}{z_{e}(x)} \frac{d z_{e}(x)}{d x}\right) \frac{\partial \eta}{\partial t} ;} \\
p^{*}=p_{*}\left(1-t^{2}\right)^{-1 / 2} ; \quad \delta_{e}^{*}=\left(1-t^{2}\right)^{3 / 4} \Delta_{e} .
\end{gathered}
$$

These transformations take into account the character of the flow in the vicinity of leading edges and the tip of the wing. For a semiinfinite wing with linear edges, such variables can reduce the problem to a $2 \mathrm{D}$ case. But in this case, because of the nonlinearity of the leading edge, the problem remains the $3 \mathrm{D}$ one. A full system of the boundary layer equations takes the following form:

$$
\begin{gathered}
\frac{\partial v_{*}}{\partial \eta}+\frac{1-t^{2}}{p_{*}}\left(z_{0} x \frac{\partial u}{\partial x}-\frac{z_{0} t x}{z_{e}(x)} \frac{\partial z_{e}(x)}{\partial x} \frac{\partial u}{\partial t}+\frac{x}{z_{e}} \frac{\partial w}{\partial t}+\frac{z_{0} u}{4}\right) \\
-\frac{t}{2 p_{*}}\left(w \frac{x}{z_{e}}-u \frac{z_{0} t x}{z_{e}(x)} \frac{d z_{e}(x)}{d x}\right)=0 ; \\
z_{0} x u \frac{1-t^{2}}{p_{*}} \frac{\partial u}{\partial x}+\frac{1-t^{2}}{p_{*}}\left(w \frac{x}{z_{e}}-\frac{t z_{0} x u}{z_{e}(x)} \frac{\partial z_{e}(x)}{\partial x}\right) \frac{\partial u}{\partial t}+v_{*} \frac{\partial u}{\partial \eta} \\
=z_{0} \frac{\gamma-1}{2 \gamma p_{*}}\left(g-u^{2}-w^{2}\right)\left(\left(1-t^{2}\right)\left(\frac{1}{2}-\frac{x}{p_{*}} \frac{\partial p_{*}}{\partial x}\right)\right. \\
\left.+\frac{t x}{z_{e}(x)} \frac{\partial z_{e}(x)}{\partial x}\left(t+\frac{1-t^{2}}{p_{*}} \frac{\partial p_{*}}{\partial t}\right)\right)+\frac{\partial}{\partial \eta}\left(N \frac{\partial u}{\partial \eta}\right) ; \\
z_{0} x u \frac{1-t^{2}}{p_{*}} \frac{\partial w}{\partial x}+v_{*} \frac{\partial w}{\partial \eta}+\frac{1-t^{2}}{p_{*}}\left(w \frac{x}{z_{e}}-u \frac{z_{0} t x}{z_{e}(x)} \frac{\partial z_{e}(x)}{\partial x}\right) \frac{\partial w}{\partial t} \\
=-\frac{\gamma-1}{2 \gamma p_{*}}\left(g-u^{2}-w^{2}\right) \frac{x}{z_{e}}\left(\frac{1-t^{2}}{p_{*}} \frac{\partial p_{*}}{\partial t}+t\right)+\frac{\partial}{\partial \eta}\left(N \frac{\partial w}{\partial \eta}\right) ; \\
z_{0} x u \frac{1-t^{2}}{p_{*}} \frac{\partial H}{\partial x}+\frac{1-t^{2}}{p_{*}}\left(w \frac{x}{z_{e}}-u \frac{z_{0} t x}{z_{e}(x)} \frac{\partial z_{e}(x)}{\partial x}\right) \frac{\partial H}{\partial t}+v_{*} \frac{\partial H}{\partial \eta} \\
=\frac{\partial}{\partial \eta}\left\{N\left[\frac{1}{\sigma} \frac{\partial H}{\partial \eta}-\frac{1-\sigma}{\sigma} \frac{\partial\left(u^{2}+w^{2}\right)}{\partial \eta}\right]\right\}
\end{gathered}
$$


where

$$
\begin{aligned}
N & =\left(H-u^{2}-w^{2}\right)^{\omega-1} \\
\Delta_{e} & =\frac{1}{p_{*}} \sqrt{\frac{\gamma-1}{2 \gamma}} \int_{0}^{\infty}\left(H-u^{2}-w^{2}\right) d \eta \\
p_{*} & =\frac{\gamma+1}{2}\left\{\frac{3}{4}\left(1-t^{2}\right) \Delta_{e}+x\left(1-t^{2}\right) \frac{\partial \Delta_{e}}{\partial x}\right. \\
& \left.+\frac{t x}{z_{e}(x)} \frac{\partial z_{e}(x)}{\partial x}\left(\frac{3}{2} \Delta_{e} t-\left(1-t^{2}\right) \frac{\partial \Delta_{e}}{\partial t}\right)\right\}^{2}
\end{aligned}
$$

The boundary conditions:

$$
\begin{aligned}
\eta=0: & u=v_{*}=w=0, H=H_{w} \\
\eta \rightarrow \infty: & u \rightarrow 1, \quad w \rightarrow 0, \quad H \rightarrow 1 .
\end{aligned}
$$

To set the boundary conditions at the trailing edge, a linear extrapolation of flow functions was used. Other conditions are the same as in the previous problem.

\section{NUMERICAL METHOD}

For the numerical solution of equations, the finite-difference method with the tridiagonal matrix algorithm $[4,5]$ is used. Central differences are used to approximate the diffusion terms. Convective terms were approximated by the second order upwind scheme [5]. To calculate the flow near boundaries, the numerical scheme is switched to the first order. To determine the displacement thickness of the boundary layer Simpson's 4th order formula is used. Because of strong changes in the functions of the flow in the process of convergence at each iteration, the underrelaxation of velocities and the enthalpy is used:

$$
f_{j, k}^{*}=(1-\alpha) f_{j, k}^{m}+\alpha f_{j, k}^{m+1}
$$

where parameter $\alpha$ is chosen empirically in such a way that there is sufficient stability and good rate of convergence ( $\alpha$ usually takes a value from 0.1 to 0.8 ). The obtained value $f_{j, k}^{*}$ is used as the initial approach in the next iteration.

The calculations showed that for stability of solution, the relaxation procedure should be also applied to pressure. The relaxation of the pressure was carried out by solving the second order differential equation [6]:

$$
\frac{d^{2} \Delta p^{n}(t)}{d t^{2}}-\alpha \Delta p^{n}(t)=\alpha\left(p^{n}(t)-p_{0}^{n}(t)\right) ; \quad \Delta p^{n}(t= \pm 1)=0 .
$$


This method can significantly accelerate the convergence of the problem and avoid nonphysical oscillations of functions.

The calculation is considered to be completed if in all cells the difference between the values of the pressure at two successive iterations becomes less than $10^{-14}$ for the edges of the wing, and less Table 1 Numerical results on successive refinement grids

\begin{tabular}{cc}
\hline Grid & $C_{p}$ \\
\hline $15 \times 100 \times 15$ & 2.14314 \\
$30 \times 200 \times 30$ & 2.42076 \\
$45 \times 300 \times 45$ & 2.54978 \\
$60 \times 400 \times 60$ & 2.62549 \\
$80 \times 500 \times 80$ & 2.67072 \\
\hline
\end{tabular}
than $10^{-5}$ for the main field. The average number of steps is approximately $20-30$ iterations on the edges, about 5000 iterations in the tip of the wing, and approximately 5000-10000 for the flow on the entire wing. Calculations on a grid of 2 million nodes consume about $30 \mathrm{~min}$ on a regular personal computer.

To estimate the real order of accuracy, the calculations on successive refinement grids were carried out (Table 1). Grids were refined from 225000 to 3200000 nodes. As the result of the calculation, the integral pressure coefficient is shown.

Using these results, it can be shown [4] that the real order of accuracy is about 1.8 . In calculations, a grid $60 \times 400 \times 60$ which produce errors about $2 \%$ was usually used.

\section{RESULTS}

Since the calculations were performed in the transformed variables, then to present the results in the physical variables the following formulas are used for the total coefficient of the surface friction (shear stress), the coefficient of surface heat fluxes, the distribution of the pressure and the distribution of the displacement thickness of the boundary layer correspondingly:

$$
\begin{array}{rlrl}
\tau_{V} & =\frac{p_{*}}{\left(1-t^{2}\right)^{3 / 4}} \frac{1}{x^{1 / 2}} \sqrt{\left(\frac{\partial u}{\partial \eta}\right)_{w}^{2}+\left(\frac{\partial w}{\partial \eta}\right)_{w}^{2}} ; & \tau_{H}=\left.\frac{p_{*}}{\left(1-t^{2}\right)^{3 / 4}} \frac{1}{x^{1 / 2}} \frac{\partial H}{\partial \eta}\right|_{w} ; \\
p^{*}=\frac{p_{*}}{\left(1-t^{2}\right)^{1 / 2}} \frac{1}{x^{3 / 4}} ; & \delta_{e}^{*}=\left(1-t^{2}\right)^{3 / 4} x^{3 / 4} \Delta_{e} .
\end{array}
$$

For numerical calculation, the following parameters were used: Prandtl number $\sigma=0.72$, the ratio of specific heats $\gamma=1.4$, the temperature factor $H_{w}=0.5$, and the linear dependence of viscosity on temperature $(N=1)$.

Figures 3 and 4 show the numerical solution of self-similar flow in cylindrical coordinate system on two wings with parameters: $(a) \Theta=135^{\circ}$ and $\beta=15^{\circ}$; and $(b) \Theta_{0}=105^{\circ}$ and $\beta=45^{\circ}$. As can be seen on wings with the point of 

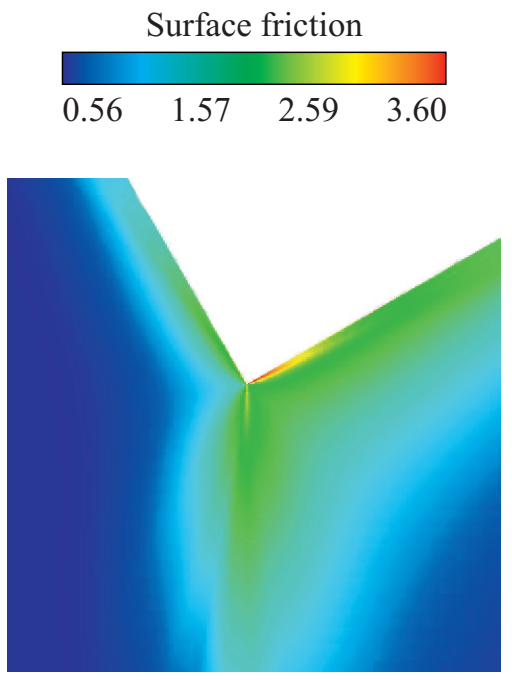

(a)
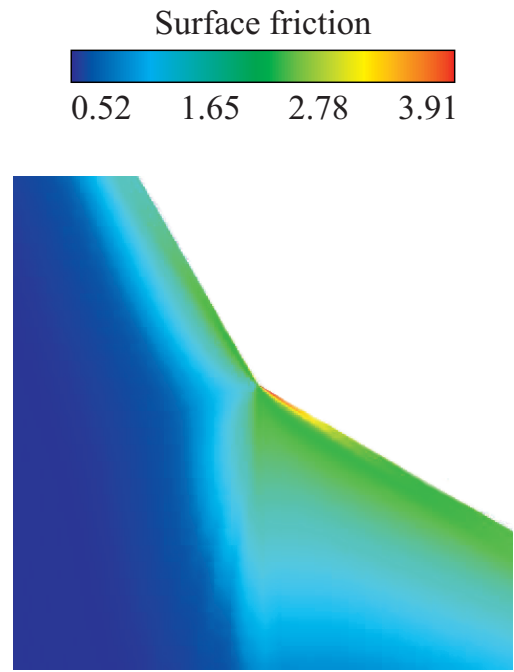

(b)

Figure 3 Surface friction near the point of inflection: $(a) \Theta=135^{\circ}$ and $\beta=15^{\circ}$; and $(b) \Theta_{0}=105^{\circ}$ and $\beta=45^{\circ}$.

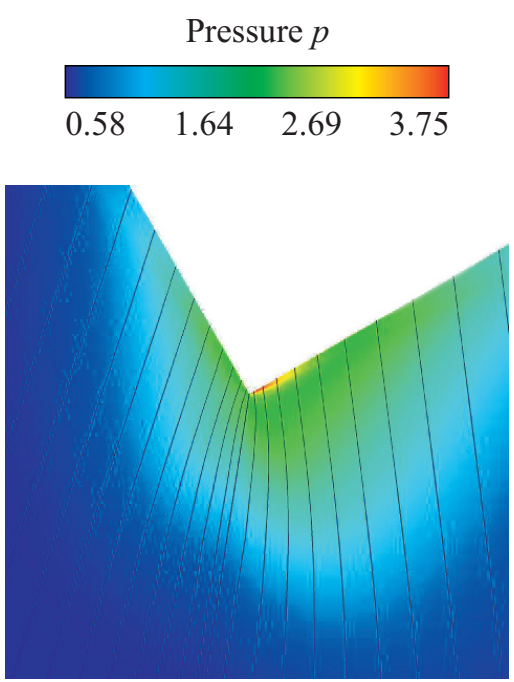

(a)

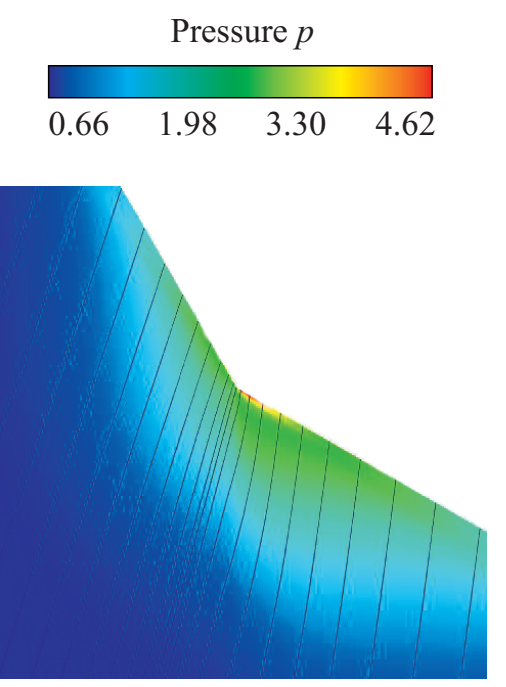

(b)

Figure 4 Pressure distribution and flowlines: (a) $\Theta=135^{\circ}$ and $\beta=15^{\circ}$; and (b) $\Theta_{0}=105^{\circ}$ and $\beta=45^{\circ}$. 


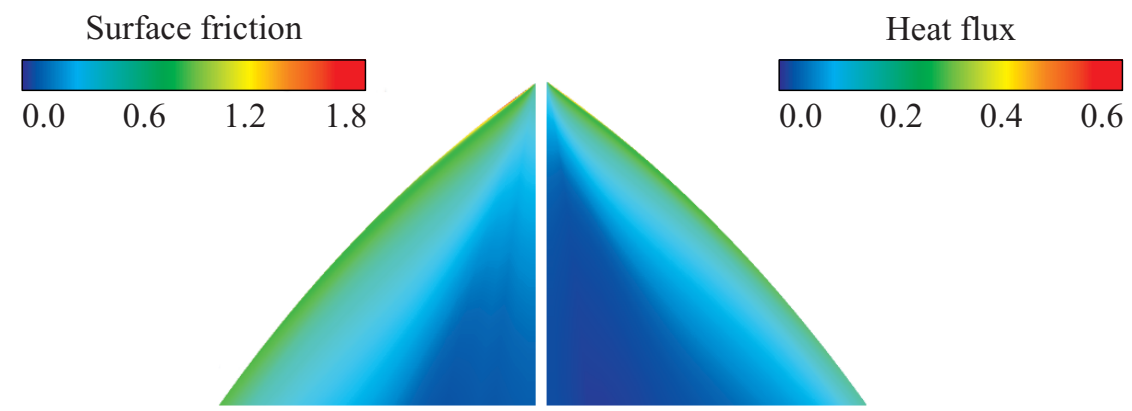

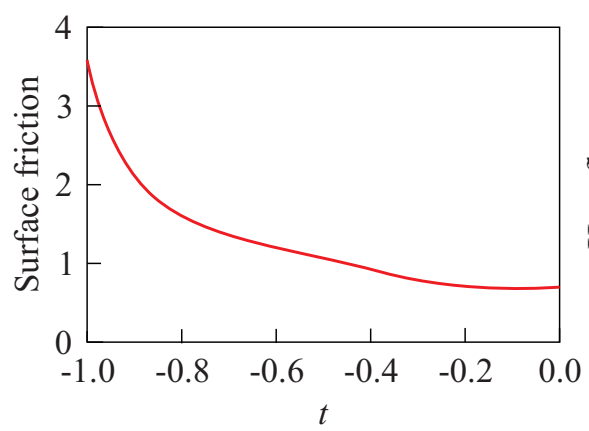

(a)

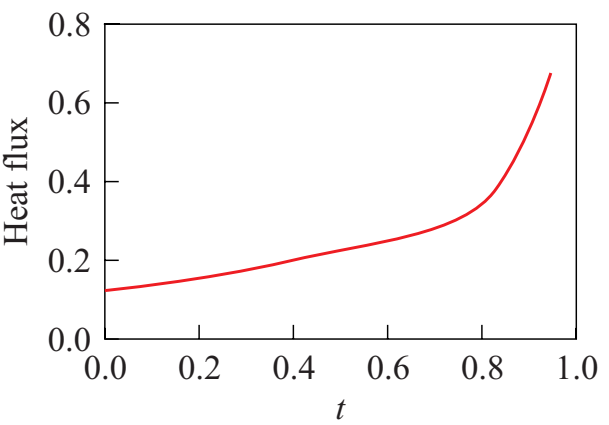

(b)

Figure 5 Distributions of surface friction $(a)$ and heat flux $(b): z_{e}(x)=\ln (x+1)$.

inflection of leading edge, there is a significant area of increased friction and heat flux in the vicinity of the critical point, and the local maximum is located in the direction of external flow. Increased pressure (see Fig. 4) is observed near the edges and the point of inflection, and for the edge with lower sweep angle, the pressure is higher. Figure 4 also shows surface flowlines.

In Fig. 5, there are shown the results of full 3D calculations and are presented distributions of the surface friction and the heat flux for a wing with a logarithmic function for the form of leading edge. Since the flow is completely symmetrical, only half of the wing is presented. Below, the corresponding distributions near the tip of the wing are shown. The maximum friction and heat fluxes are located on the edges near the tip of the wing. There is a good qualitative agreement between the distributions of the friction and the heat flux.

Figure 6 shows the pressure distribution and surface streamlines for the same wing. As can be seen, the maximum pressure is located near the tip of the wing and near leading edges. In this case, the streamlines smoothly approach the plane of symmetry, where there is a local increase in pressure. It is worth noting that the flow lines are almost parallel in the entire region of high pressure. In 


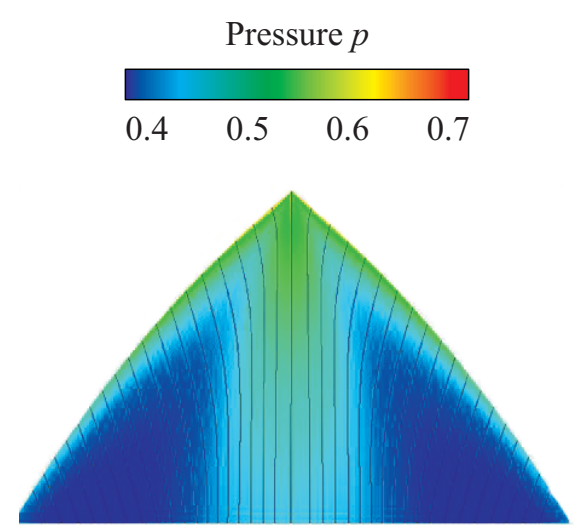

(a)

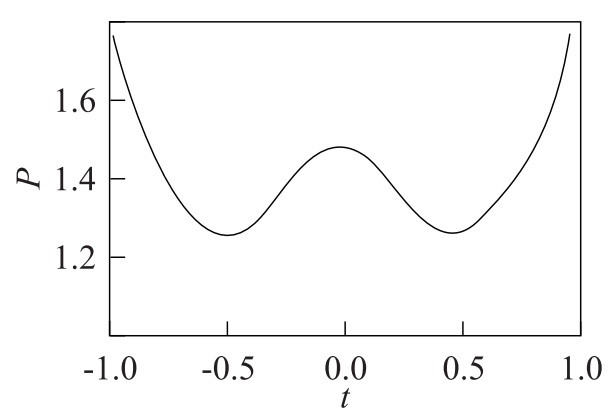

(b)

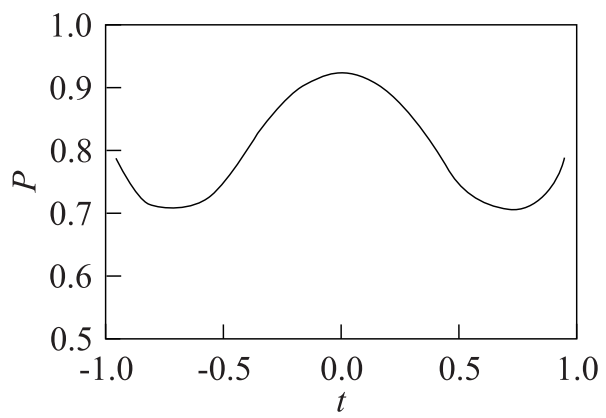

(c)

Figure 6 Pressure distribution $(a)$ and surface flow lines $(x=0(b)$ and $x=1(c))$ : $z_{e}(x)=\ln (x+1)$.

Figs. $6 b$ and $6 c$, there are shown the pressure distributions along the transverse coordinate at the top of the wing $(x=0)$ and at the trailing edge $(x=1)$. As can be seen, with increasing $x$, the pressure is lowered. At the leading edge, it is much more substantial (reduction of almost 3 times) than in the symmetry plane, where it falls only one third.

In Fig. 7, the distributions of the surface friction and the heat flux are presented for the case of another wing with a form of leading as a function of $(x / 2) \exp (x)$. As before, there is a qualitative correspondence between the friction and heat flux. In Fig. $7 a$, the flow lines are also shown, which, unlike the previous wing, are all flocking to the plane of symmetry.

The distribution of the pressure is shown in Fig. 8. The maximum value is achieved at the leading edge near the trailing edge but not near the vertex of the wing as in the previous case. In the symmetry plane near the tip, a local increase in the pressure with approaching the rear edge almost disappears. As mentioned above, for the previous wing, increasing of the pressure in the plane of symmetry becomes more pronounced near trailing edge. 


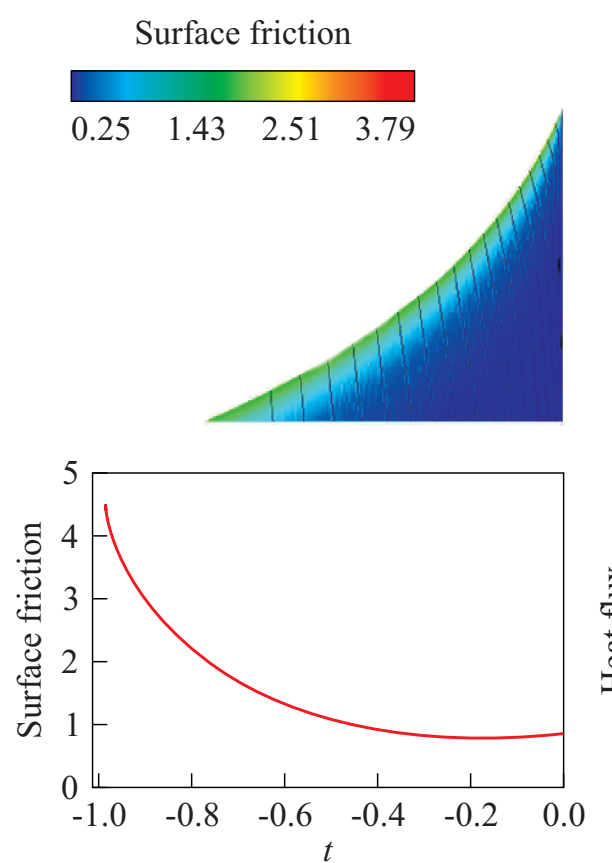

(a)
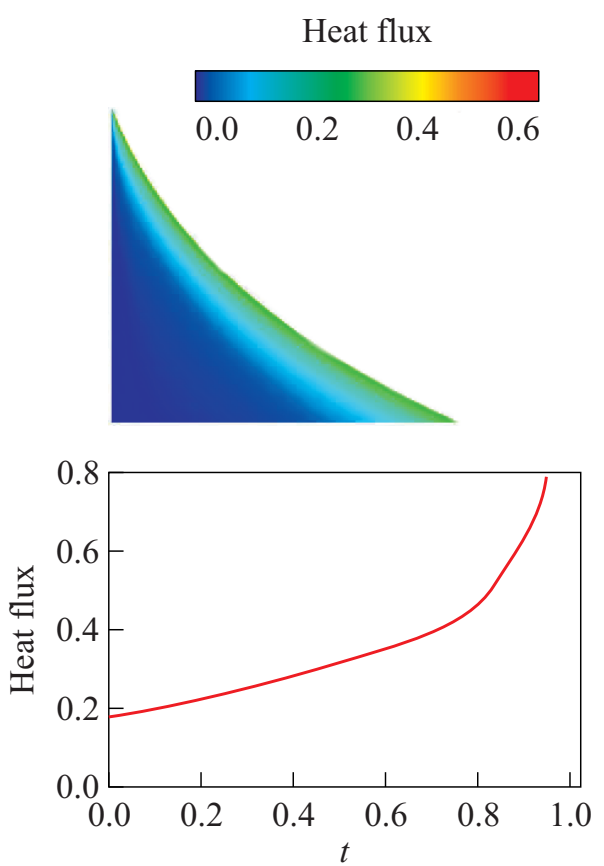

(b)

Figure 7 Surface friction $(a)$ with flow lines and heat flux $(b): z_{e}(x)=(x / 2) \exp (x)$.

For a wing with logarithmic edges, the calculations were made for temperature factors 0.1 and 0.9 . Figure $9 a$ shows the pressure distribution. Heating of the surface of the wing not only leads to a 3-time increase in pressure, but also to a more pronounced maximum in the plane of symmetry. In Fig. $9 b$, the distribution of the surface friction near the tip of the wing $(x=0)$ is shown. As can be seen, the heating of the surface from $H_{w}=0.1$ to 0.9 leads to an increase in friction of about 2.5 times.

\section{CONCLUDING REMARKS}

1. The boundary problems for the flow in the vicinity of the point of inflection and on a wing with a variable form of leading edge are formulated.

2. The method developed can be easily applied to the investigation of the boundary layer on a wing of a complex shape with sharp leading edges at zero angle of attack. 


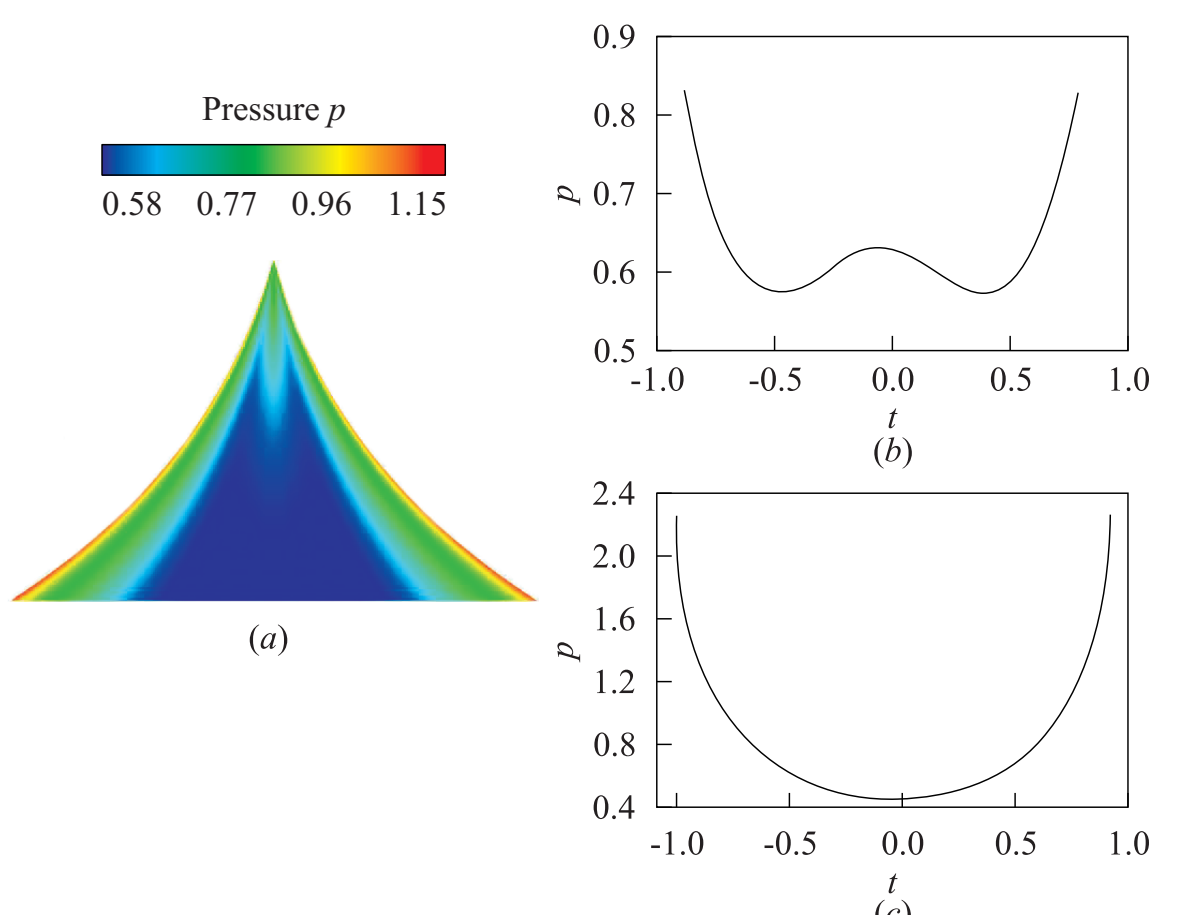

(c)

Figure 8 Pressure distribution $(a)$ and surface flow lines $(x=0(b)$ and $x=1(c))$ : $\left.z_{e}(x)=(x / 2) \exp (x)\right)$.

3. It is shown that local increase in the heat flux, the surface friction and the pressure can be produced by the point of inflection of leading edge.

\section{ACKNOWLEDGMENTS}

The work was partially supported by the Russian Foundation for Basic Research (Grant No. 10-01-00173-a).

\section{REFERENCES}

1. Hayes, W. D., and R. F. Probstein. 1966. Hypersonic flow theory. New York: Academic Press.

2. Neiland, V. Ya., V. V. Bogolepov, G. N. Dudin, and I. I. Lipatov. 2008. Asymptotic theory of supersonic viscous gas flows. Amsterdam-London-New York: Elsevier. 


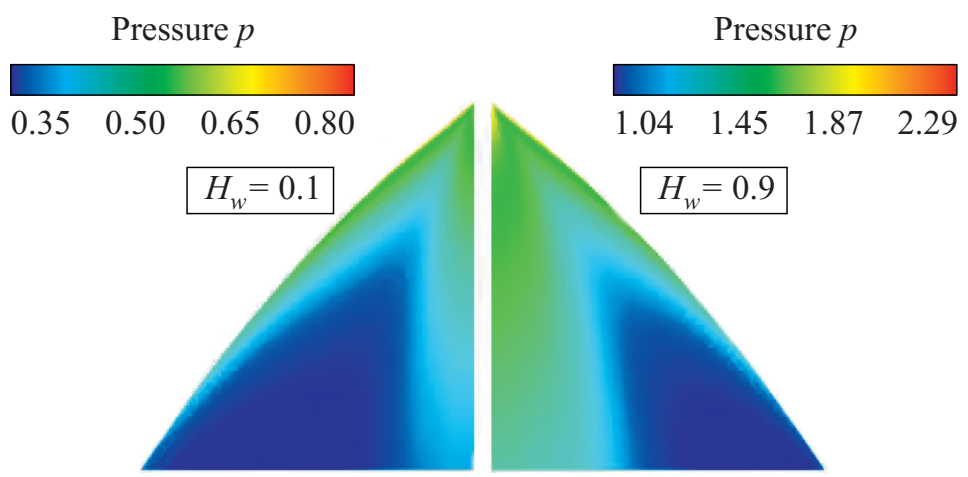

(a)

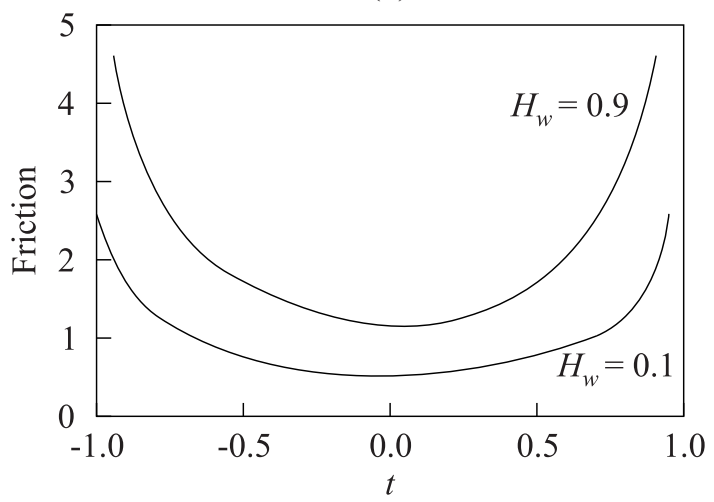

(b)

Figure 9 Pressure distributions $(a)$ and surface friction with different temperature factors $\left.(b): z_{(} x\right)=\ln (x+1)$. (Refer color plate, p. XL.)

3. Anderson, J. D. 1989. Hypersonic and high temperature gas dynamics. McGraw-Hill Book Co.

4. Ferziger, J. H., and M. Peric. 2001. Computational methods for fluid dynamics. 3rd ed. Springer. $431 \mathrm{p}$.

5. Bashkin, V. A., and G. N. Dudin. 2006. Theory of hypersonic viscous flows. Moscow: MPhTI. [In Russian.]

6. Dudin, G. N., and D. O. Lyzhin. 1983. A method of calculation a regime of strong viscous interaction on a delta wing. Fluid Dyn. 18:596-601. 